Volume 70, Number 2, Pages 762-772(2021)

DOI: $10.31801 /$ cfsuasmas. 851539

ISSN 1303-5991 E-ISSN 2618-6470

Received by the editors: January 1, 2021; Accepted: March 12, 2021

\title{
A NOTE ON THE GENERATING SETS FOR THE MAPPING
} CLASS GROUPS

\author{
Elif DALYAN ${ }^{1}$ and Elif MEDETOĞULLARI ${ }^{2}$ \\ ${ }^{1}$ Department of Mathematics, Hitit University, Çorum, TURKEY \\ ${ }^{2}$ Department of Mathematics and Science Education, TED University, Ankara, TURKEY
}

\begin{abstract}
In this short note, we obtain generating sets with two elements for the mapping class group of closed, oriented surfaces of genus three and four, containing elements of the lowest order known so far.
\end{abstract}

\section{INTRODUCTION}

Let $\Sigma_{g}$ denote a closed, oriented surface of genus $g$. Let $\mathrm{MCG}\left(\Sigma_{g}\right)$ denote the group of isotopy classes of orientation preserving self homeomorphisms, which is called the mapping class group of the surface $\Sigma_{g}$.

Dehn, 1 proved that the mapping class group of a compact, closed surface $\Sigma_{g}$, $\operatorname{MCG}\left(\Sigma_{g}\right)$, is generated by twists, which are now called Dehn twists. Lickorish 6], unaware of Dehn's work, proved that the group is generated by $3 \mathrm{~g}-1$ Dehn twists about the non-separating curves that are given in Figure 1

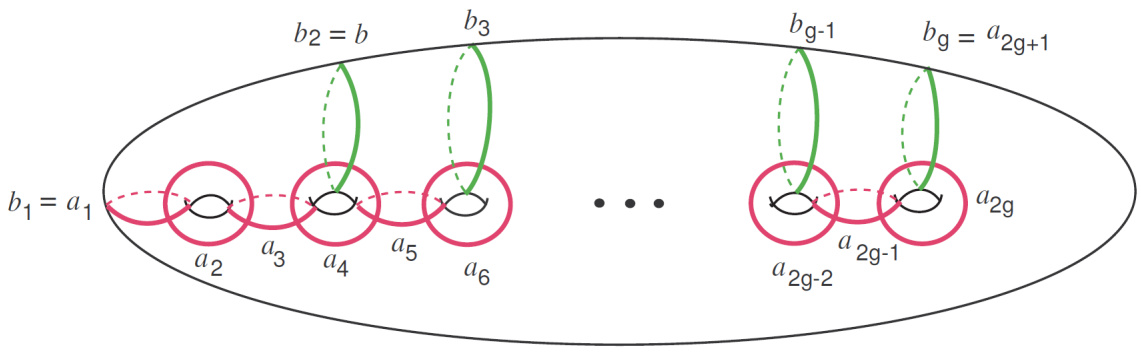

FIGURE 1. Lickorish generators

2020 Mathematics Subject Classification. Primary 57M07; Secondary 20F38.

Keywords and phrases. Mapping class groups, generators, small order, Dehn twists.

घlifdalyan@hitit.edu.tr-Corresponding author; elif.medet@gmail.com

(D) 0000-0003-2099-7584; 0000-0002-6196-8184. 
Following these developments, Humphries 3 showed that, one can obtain the Dehn twists about the non-seperating curves $b_{3}, b_{4}, \ldots, b_{g}$, from the remaining collection of Lickorish generators, see Figure 1. Hence, Humphries proved that $2 \mathrm{~g}+1$ of Lickorish generators are sufficient to generate the $\operatorname{MCG}\left(\Sigma_{g}\right)$. These generators are the Dehn twists about the non-seperating curves of Figure 2 In the same paper he showed that this number is in fact minimal.

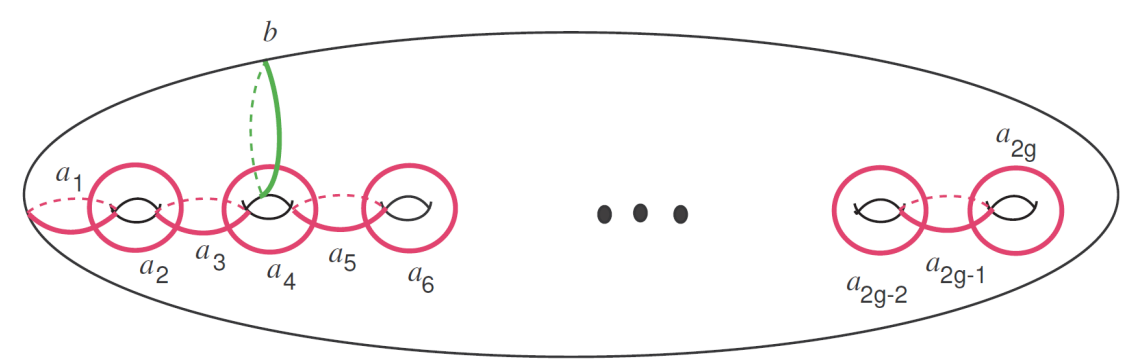

FIGURE 2. Humphries generators

These generators are of infinite order, hence the next question that comes to minds: Can we have generators of finite order? Let $A_{i}$ and $B_{i}$ denote Dehn twist about simple closed curves $a_{i}$ and $b_{i}$ given in Figure 3, respectively Wajnryb 7 proved that MCG $\left(\Sigma_{g}\right)$ is generated by $\left\langle S, B_{g-1} B_{g}^{-1}\right\rangle$, where $S=A_{2 g} A_{2 g-1} \cdots A_{2} A_{1}$, and is of order $4 g+2$, see Figure 3

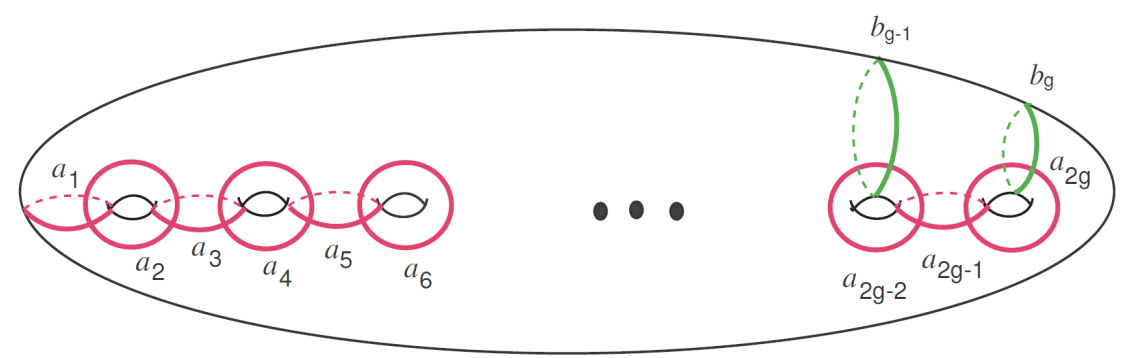

FiguRE 3. Wajnryb generators

Then Korkmaz improved this result, by the following theorem:

Theorem 1. [4] Suppose that $g \geq 2$ and $\Sigma_{g}$ is a closed oriented surface of genus g. The mapping class group $\mathrm{MCG}\left(\Sigma_{g}\right)$ of $\Sigma_{g}$ is generated by $S$ and $B$. 
In Theorem 1. Korkmaz proved that MCG $\left(\Sigma_{g}\right)$ of a closed surface $\Sigma_{g}$ is generated by two elements. One of the generators is $\mathrm{B}$, Dehn twist about the curve $b$, hence it is of infinite order. The other generator is $S=A_{2 g} A_{2 g-1} \cdots A_{2} A_{1}$ and is of order $4 g+2$, see Figure 2 for the curves $b, a_{1}, a_{2}, \ldots, a_{2 g}$. In this note we lower this order using the homeomorphism $Q=A_{2 g+1} A_{2 g} \cdots A_{2} A_{1}$ for a genus 4 surface and $Q^{\prime}=A_{2 g+1} A_{2 g} \cdots A_{2} A_{1} A_{1}$ for a genus 3 surface. In the following theorem we show that MCG $\left(\Sigma_{4}\right)$ is generated by $\langle Q, B\rangle$ where $Q=A_{9} A_{8} \cdots A_{2} A_{1}$, and is of order 10 ( that is $2 g+2$ for $g=4$ ).

Theorem 2. Mapping class group of a closed, oriented genus 4 surface is generated by $Q$, an element of order 10, and the Dehn twist B.

Our second result gives even a lower order generator $Q^{\prime}=A_{7} A_{6} \cdots A_{2} A_{1} A_{1}$, which is of order 7 (that is $2 g+1$ for $g=3$ ), for the group of mapping classes of the closed genus 3 surface:

Theorem 3. Mapping class group of a closed, oriented genus 3 surface is generated by $Q^{\prime}$, an element of order 7 , and the Dehn twist B.

Remark 4. Even though we tried to generalize Theorem 2 and Theorem 3 for higher genus surfaces, it was not possible with the current technique. One needs other approaches to prove such generalizations.

\section{Preliminaries}

In the next section, we will prove Theorem 2 and Theorem 3 using some basic properties of the group of mapping classes. In this section we will review the basic properties that we need, without giving their proofs. For the proofs see [2], 5].

Convention: In this note we consider simple closed curves and homeomorphisms up to isotopy and the usual composition of functions, meaning that, if there are several number of functions (Dehn twists) to be composed, we first apply the function on the right then continue from right to left. Throughout the paper, when we use an equality sign between the curves or homeomorphisms, we mean the equivalence up to isotopy.

Notation: We use lower case letters $\left(a_{i}, b, b_{j}, \ldots\right)$ for the simple closed curves, and capital letters $\left(A_{i}, B, B_{j}, \ldots\right)$ to denote the Dehn twists about these curves $\left(a_{i}, b, b_{j}, \ldots\right)$.

Relations:

(1) Let $c, d$ be two simple closed curves on an oriented surface $\Sigma_{g}$ and let $H$ be an orientation preserving self homeomorphism of the surface such that $H(c)=d$. Then

$$
H C H^{-1}=D \text {. }
$$

(2) Commutativity relation: Let $c, d$ be two disjoint simple closed curves on an oriented surface $\Sigma_{g}$, then the Dehn twists around the curves $c$ and $d$ commute:

$$
C D=D C
$$




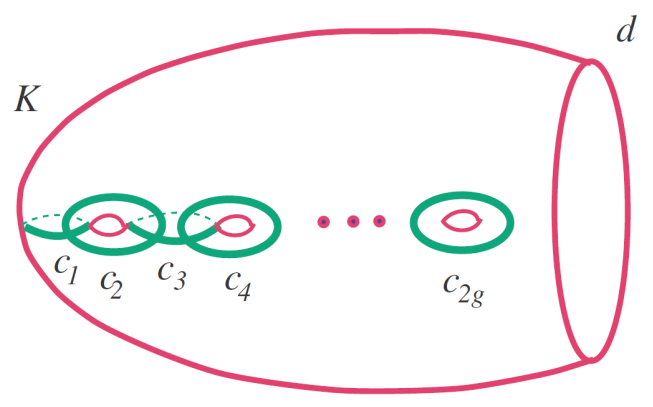

FiguRE 4. $K$, the neighborhood of union of even number of curves in the chain

(3) Braid relation: Let $c, d$ be two simple closed curves, intersecting transversely at one point, on an oriented surface $\Sigma_{g}$, then the Dehn twists around the curves $c$ and $d$ satisfy the relation below:

$$
C D C=D C D
$$

\section{(4) Chain relation:}

First we define the chain, then we will give the chain relation.

Definition 5. Chain: Let $c_{1}, c_{2}, \ldots, c_{n}$ be a sequence of simple closed curves on an orientable surface. If only the consecutive ones intersect transversely at one point, and the others are disjoint, then this sequence of simple closed curves $c_{1}, c_{2}, \ldots, c_{n}$ is called a chain.

Let $K$ be a tubular neighborhood of union of curves in the chain. There are two cases according to the parity of number of curves ( $\mathrm{n}$ is even or odd) in the chain:

- If $n=2 g$, then $K$ is an orientable genus $g$ surface of one boundary component, call that boundary component $d$, see Figure 4 .

- If $n=2 g+1$, then $K$ is an orientable genus $g$ surface of two boundary components, call them $d_{1}$ and $d_{2}$.

Then we have the following relations, which are called the chain relations in $\operatorname{MCG}(K)$ :

- If $n=2 g$, then we have $\left(C_{1} C_{2} \cdots C_{2 g}\right)^{4 g+2}=D$

- If $n=2 g+1$, then we have $\left(C_{1} C_{2} \cdots C_{2 g+1}\right)^{2 g+2}=D_{1} D_{2}$.

\section{Proofs}

We will start this section with the proof of Theorem 2, In Theorem 2, we show that $\operatorname{MCG}\left(\Sigma_{4}\right)=<Q, B>$, where $Q=A_{9} A_{8} \cdots A_{2} A_{1}$, see Figure 1 for the curves $a_{1}, a_{2}, \ldots, a_{9}$. Then we show that the order of the element $Q$ is 10 , in Lemma 8 . 


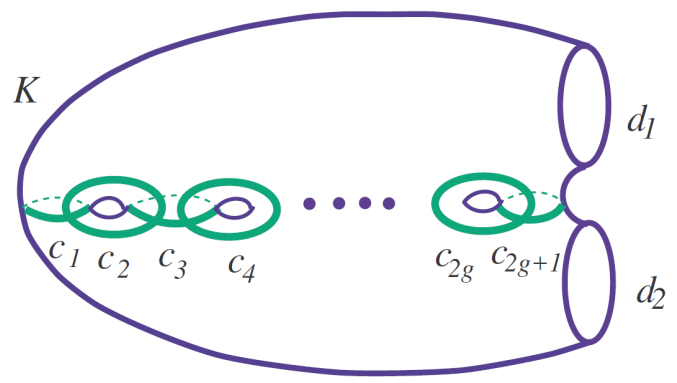

FiguRE 5. $K$, the neighborhood of union of odd number of curves in the chain

Hence, it is a lower order element than the one in Theorem 1, in which Korkmaz proved that $\operatorname{MCG}\left(\Sigma_{4}\right)=<S, B>$ and $S=A_{8} A_{7} \cdots A_{2} A_{1}$. The element $S$ is of order 14.

In order to prove these results we need some preparatory results. Let $G=<$ $Q, B>$ be the subgroup of $\operatorname{MCG}\left(\Sigma_{4}\right)$ generated by $Q$ and $B$. Let $C_{1}, C_{2}, C_{3}$, $D_{1}, D_{2}, E_{1}, E_{2}, F_{1}$ and $B_{3}$ be the Dehn twists around the curves $c_{1}, c_{2}, c_{3}, d_{1}, d_{2}$, $e_{1}, e_{2}, f_{1}$ and $b_{3}$, respectively, which are shown in Figure 6 .

Lemma 6. The Dehn twists $C_{1}, C_{2}, C_{3}, D_{1}, D_{2}, E_{1}, E_{2}, F_{1}$ and $B_{3}$ are contained in $G=<Q, B>$.

Proof of Lemma 6. We will show that we can get the Dehn twists $C_{1}, C_{2}, C_{3}, D_{1}, D_{2}$, $E_{1}, E_{2}, F_{1}, B_{3}$ from the homeomorphisms $Q$ and $B$ by applying the Relation (1) several times. First, applying the homeomorphism $Q^{-1}$ to the simple closed curve $b$, we get the simple closed curve $c_{1}$. Hence, we have

- $Q^{-1}(b)=c_{1}$ and by Relation (1), we can write $C_{1}=Q^{-1} B Q$. Since $Q$ and $B$ are already in the group $G$, we conclude that $C_{1} \in G$.

Similarly, we repeat this process:

- $Q^{-1}\left(c_{1}\right)=d_{1}$, using Relation (1) and since $C_{1}, Q \in G$ we have $D_{1} \in G$.

- $Q^{-1}\left(d_{1}\right)=c_{2}$ which implies $C_{2} \in G$.

- $Q^{-1}\left(c_{2}\right)=d_{2}$ which implies $D_{2} \in G$.

- $Q^{-1}\left(d_{2}\right)=c_{3}$ which implies $C_{3} \in G$.

- $Q^{-1}\left(c_{3}\right)=b_{3}$ which implies $B_{3} \in G$.

- $Q^{-1}\left(b_{3}\right)=e_{1}$ which implies $E_{1} \in G$.

- $Q^{-1}\left(e_{1}\right)=f_{1}$ which implies $F_{1} \in G$.

- $Q^{-1}\left(f_{1}\right)=e_{2}$ which implies $E_{2} \in G$. 


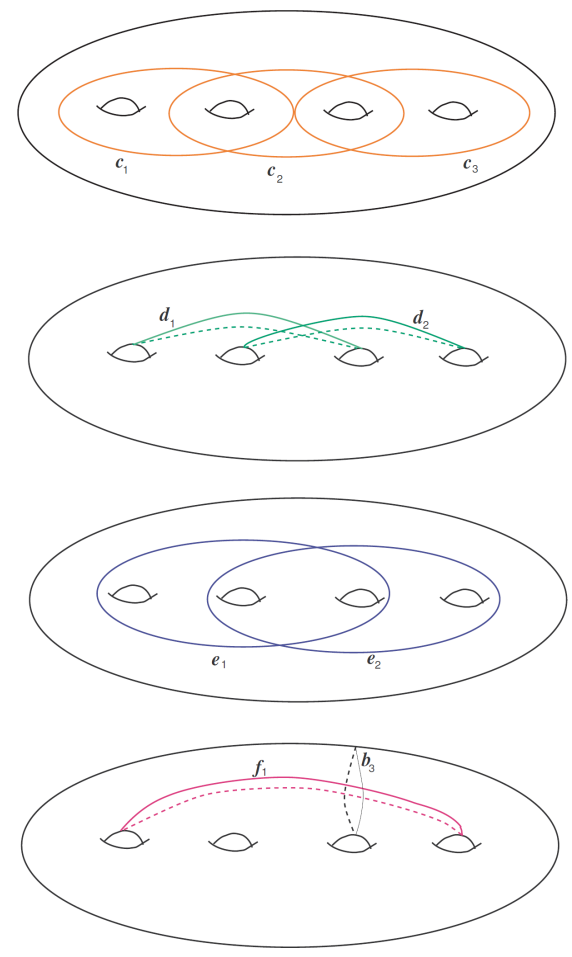

Figure $6 . c_{1}, c_{2}, c_{3}, d_{1}, d_{2}, e_{1}, e_{2}, f_{1}, b_{3}$, curves on a genus 4 surface

The main idea of the proof of Theorem 2 is to show that the $G$-orbit of the curve $b$ contains the simple closed curves $a_{1}, a_{2}, \ldots, a_{8}$. As a result of this, all the Humphries generators $A_{1}, A_{2}, \ldots, A_{8}$ and $B$ of $\operatorname{MCG}\left(\Sigma_{4}\right)$ are contained in $G$, hence we conclude that $\operatorname{MCG}\left(\Sigma_{4}\right)=G$.

Proof of Theorem 2. Let $h$ be the self homeomorphism of the surface $\Sigma_{4}$ which is given by the product of the Dehn twists: $C_{2} D_{1} B B_{3}^{-1}$. We will apply the homeomorphism $h$ to the curve $c_{2}$, and obtain the non-separating simple closed curve $a_{3}$, which is shown in detail in Figure 7. Using Relation (1) and Lemma 6, we can say that the Dehn twist $A_{3}$ is in $G$. Then applying the homeomorphism $Q^{-1}$ to the curve $a_{3}$, we get:

- $Q^{-1}\left(a_{3}\right)=a_{4}$, using Relation (1) we say that $Q^{-1} A_{3} Q=A_{4}$ and since $Q, A_{3} \in G$ we have $A_{4} \in G$. Using repeatedly this process, we get the following:

- $Q^{-1}\left(a_{4}\right)=a_{5}$ which implies that $A_{5} \in G$.

- $Q^{-1}\left(a_{5}\right)=a_{6}$ which implies that $A_{6} \in G$. 


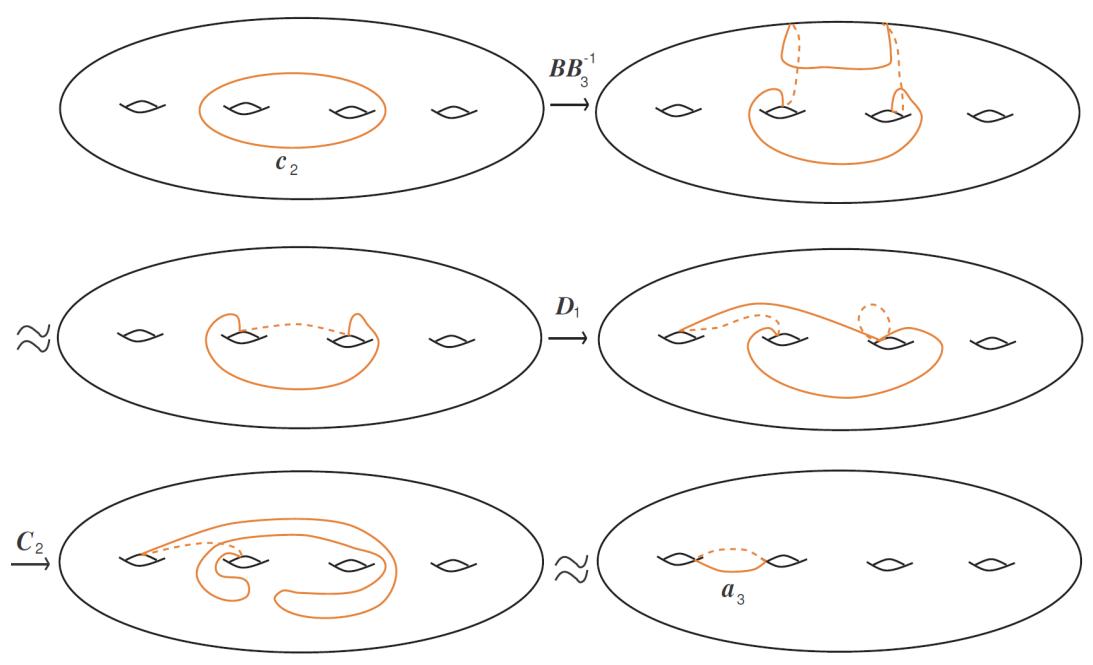

FIGURE 7. $h\left(c_{2}\right)=a_{3}$

- $Q^{-1}\left(a_{6}\right)=a_{7}$ which implies that $A_{7} \in G$.

- $Q^{-1}\left(a_{7}\right)=a_{8}$ which implies that $A_{8} \in G$.

Similarly applying the homeomorphism $Q$ and $Q^{2}$ to the curve $a_{3}$, we get $a_{2}$ and $a_{1}$ :

- $Q\left(a_{3}\right)=a_{2}$ which implies that $A_{2} \in G$.

- $Q^{2}\left(a_{3}\right)=a_{1}$ which implies that $A_{1} \in G$.

Therefore, we get the result that all Humphries generators of $\operatorname{MCG}\left(\Sigma_{4}\right)$ are contained in $G$, and hence, conclude that $G=\operatorname{MCG}\left(\Sigma_{4}\right)$.

Now we are going to prove Theorem 3, which implies that $\operatorname{MCG}\left(\Sigma_{3}\right)=\left\langle Q^{\prime}, B\right\rangle$, where $Q^{\prime}=A_{7} A_{6} A_{5} A_{4} A_{3} A_{2} A_{1} A_{1}$. The idea in the proof of Theorem 3 is similar to the one in the proof of Theorem 2. In order to prove Theorem 3 , we need the following lemma. For the simple closed curves that we use in Lemma 7 , see Figure 8 .

Lemma 7. The Dehn twists $C_{1}, C_{2}, D_{1}$ are in the group $G^{\prime}=\left\langle Q^{\prime}, B\right\rangle$.

Proof of Lemma 7. We start with the simple closed curve $b$, and apply the homeomorphism $Q^{\prime}$. We get $Q^{\prime}(b)=c_{2}$ and by Relation (1) we have, $C_{2}=Q^{\prime} B Q^{\prime-1}$. Since $Q^{\prime}$ and $B$ are in the group $G^{\prime}$, we deduce that $C_{2} \in G^{\prime}$.

Similarly,

$Q^{\prime}\left(c_{2}\right)=d_{1}$ which implies that $D_{1} \in G^{\prime}$.

$Q^{\prime}\left(d_{1}\right)=c_{1}$ which implies that $C_{1} \in G^{\prime}$. 


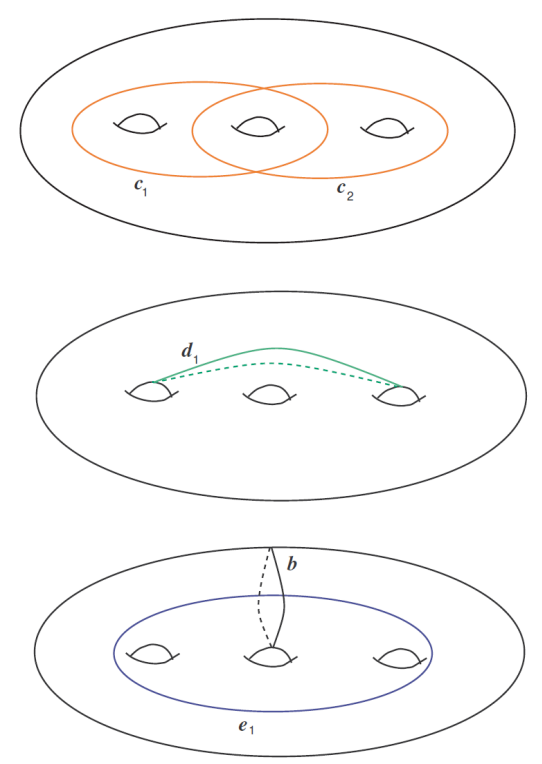

Figure 8. $c_{1}, c_{2}, d_{1}, e_{1}, b$ curves on a genus 3 surface

Proof of Theorem 3. Let $\mathrm{h}$ be the self-homeomorphism of the genus 3 surface to itself, given by $h=C_{1}^{-1}\left(Q^{\prime}\right)^{-1}$. We apply the homeomorphism $h$ to the simple closed curve $b$, and see that $h(b)=a_{1}$, as in Figure 9. Using Relation (1), we get that $A_{1} \in\left\langle Q^{\prime}, B\right\rangle=G^{\prime}$. Note that for a genus 3 surface, the homeomorphism $Q$ is given by $Q=A_{7} A_{6} A_{5} A_{4} A_{3} A_{2} A_{1}$ and $Q=Q^{\prime} A_{1}^{-1}$, hence, $Q \in G^{\prime}$.

- $Q^{-1}\left(a_{1}\right)=a_{2}$, using Relation (1) we say that $Q^{-1} A_{1} Q=A_{2}$ and since $Q$, $A_{1} \in G^{\prime}$ we have $A_{2} \in G^{\prime}$. Upon repeating this process we get the following results:

- $Q^{-1}\left(a_{2}\right)=a_{3}$ which implies that $A_{3} \in G^{\prime}$.

- $Q^{-1}\left(a_{3}\right)=a_{4}$ which implies that $A_{4} \in G^{\prime}$.

- $Q^{-1}\left(a_{4}\right)=a_{5}$ which implies that $A_{5} \in G^{\prime}$.

- $Q^{-1}\left(a_{5}\right)=a_{6}$ which implies that $A_{6} \in G^{\prime}$.

Therefore all the Humphries generators of $\operatorname{MCG}\left(\Sigma_{3}\right)$, are contained in $G^{\prime}$, hence, we conclude that $\operatorname{MCG}\left(\Sigma_{3}\right)=G^{\prime}$.

Lemma 8. The order of the element $Q=A_{9} A_{8} \cdots A_{2} A_{1}$ is 10 .

Proof of Lemma 8. The curves defining $Q$ form a chain on a closed genus 4-surface $\Sigma_{4}$. Then using the chain relation we get, $Q^{10}=i d$. Hence the order of $Q$ is at 


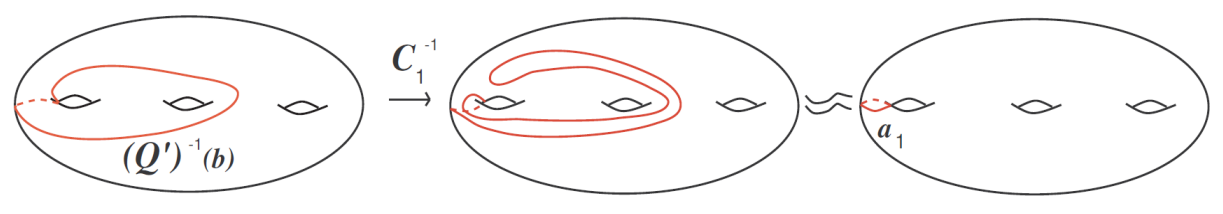

FiguRE $9 . h(b)=a_{1}$

most 10. Now, take the simple closed curve $e_{2}$ on $\Sigma_{4}$, see Figure6. From the Proof of Lemma 6 , we observe that $Q^{i}\left(e_{2}\right) \neq e_{2}$ for $1 \leq i \leq 9$. Therefore the order of $Q$ is 10 .

Lemma 9. The order of the element $Q^{\prime}=A_{7} A_{6} \cdots A_{2} A_{1} A_{1}$ is 7 .

Proof of Lemma 9. Using commutativity and braid relation, we have $\left(Q^{\prime}\right)^{7}=Q^{8}$, where $Q=A_{7} A_{6} A_{5} A_{4} A_{3} A_{2} A_{1}$ on a closed genus 3 -surface $\Sigma_{3}$ :

$$
\begin{aligned}
\left(Q^{\prime}\right)^{7} & =A_{7} A_{6} A_{5} A_{4} A_{3} A_{2} A_{1} A_{1}\left(Q^{\prime}\right)^{6} \\
& =(Q) A_{1}\left(Q^{\prime}\right)^{6} \\
& =(Q) A_{1}\left(A_{7} A_{6} A_{5} A_{4} A_{3} A_{2} A_{1} A_{1}\right)\left(Q^{\prime}\right)^{5} \\
& =(Q)^{2} A_{2} A_{1}\left(Q^{\prime}\right)^{5} \\
& =(Q)^{3} A_{3} A_{2} A_{1}\left(Q^{\prime}\right)^{4} \\
& =(Q)^{4} A_{4} A_{3} A_{2} A_{1}\left(Q^{\prime}\right)^{3} \\
& =(Q)^{5} A_{5} A_{4} A_{3} A_{2} A_{1}\left(Q^{\prime}\right)^{2} \\
& =(Q)^{6} A_{6} A_{5} A_{4} A_{3} A_{2} A_{1}\left(Q^{\prime}\right) \\
& =(Q)^{7} A_{7} A_{6} A_{5} A_{4} A_{3} A_{2} A_{1} \\
& =(Q)^{8}
\end{aligned}
$$

Moreover since $Q$ is a chain on a closed $\Sigma_{3}$ surface, from the chain relation we have, $Q^{8}=i d$, which implies that, $\left(Q^{\prime}\right)^{7}=Q^{8}=i d$, hence, the order of $Q^{\prime}$ is at most 7 .

On the other hand, one can easily check that $\left(Q^{\prime}\right)^{i}\left(a_{7}\right) \neq a_{7}$ for $1 \leq i \leq 6$ which is shown in the Figure 10. Therefore the order of $Q^{\prime}$ is 7 . 


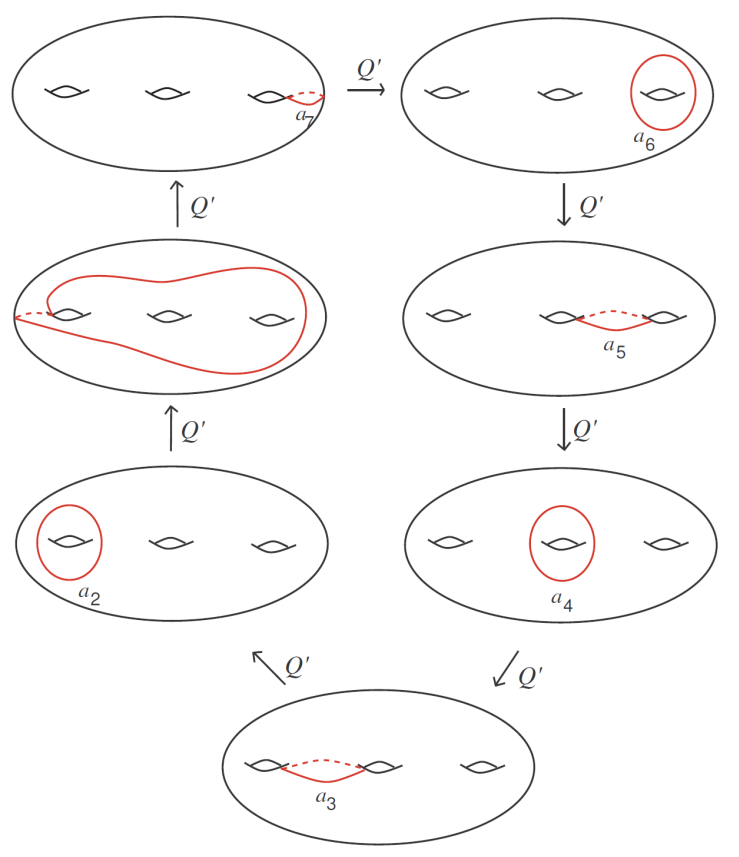

Figure 10. $\left(Q^{\prime}\right)^{i}\left(a_{7}\right) \neq a_{7}$ for $1 \leq i \leq 6$

Authors Contribution Statement The authors contributed equally. All authors read and approved the final copy of the manuscript.

Declaration of Competing Interests The authors declare that they have no competing interests.

Acknowledgement The authors would like to thank Mustafa Korkmaz and the referees for their valuable suggestions which helped to improve the manuscript.

\section{REFERENCES}

[1] Dehn, M., Die Gruppe der Abbildungsklassen, Acta Math. 69 (1938), 133-206. https://doi.org/10.1007/BF02547712

[2] Farb, B., Margalit, D., A primer on mapping class groups, Princeton University Press, (2011).

[3] Humphries, S. P., Generators for the mapping class group, In: Topology of low-dimensional manifolds (Proc. Second Sussex Conf., Chelwood Gate, 1977), Lecture Notes in Math., 722, Springer, (1979), 44-47. https://doi.org/10.1007/BFb0063188

[4] Korkmaz, M., Generating the surface mapping class group by two elements, Trans. Amer. Math. Soc. 357 (2005), 3299-3310. https://doi.org/10.1090/S0002-9947-04-03605-0 
[5] Korkmaz, M., Minimal generating sets for the mapping class group of a surface, Handbook of Teichmüller Theory 3 (2012), 441-463.

[6] Lickorisch, W. B. R., A finite set of generators for the homeotopy group of a 2-manifold, Proc. Cambridge Philos. Soc. 60 (1964), 769-778. https://doi.org/10.1017/S030500410003824X

[7] Wajnryb, B., Mapping class group of a surface is generated by two elements, Topology 35, No. 2 (1996), 377-383. https://doi.org/10.1016/0040-9383 